\title{
Proteomic analysis of human umbilical cord serum exosomes using mass spectrometry and preliminary study of their biological activities in liver cancer cell lines
}

\author{
DONGLIE ZHU ${ }^{1,2^{*}}$, WENHUI LI ${ }^{3 *}$, CHENG FANG $^{4 *}$, RUOZHE YIN $^{5}$, \\ MINGZUO JIANG ${ }^{6}, \mathrm{XING} \mathrm{LV}^{7}$ and YONG CHEN ${ }^{1}$
}

\begin{abstract}
${ }^{1}$ Department of Hepatobiliary Surgery, Xijing Hospital, Fourth Military Medical University, Xi'an, Shaanxi 710032; ${ }^{2}$ Department of General Surgery, The Air Force Hospital of Northern Theater of People's Liberation Army of China, Shenyang, Liaoning 110042; ${ }^{3}$ Department of Gynecology and Obstetrics, Changhai Hospital; ${ }^{4}$ Department of Hepatobiliary Surgery, Eastern Hepatobiliary Surgery Hospital, Second Military Medical University, Shanghai 200438; ${ }^{5}$ Department of Emergency, The Second Affiliated Hospital of Xi'an Jiaotong University, Xi'an, Shaanxi 710004; ${ }^{6}$ Department of Gastroenterology and Hepatology, Jinling Hospital, Medical School of Nanjing University, Nanjing, Jiangsu 210002; ${ }^{7}$ Department of Respiratory Medicine, Xijing Hospital, Fourth Military Medical University, Xi'an, Shaanxi 710032, P.R. China
\end{abstract}

Received December 20, 2020; Accepted July 1, 2021

DOI: $10.3892 /$ etm.2021.10966

\begin{abstract}
Exosomes are membranous extracellular vesicles 50-100 $\mathrm{nm}$ in size, which are involved in cellular communication via the delivery of proteins, lipids and RNA. Emerging evidence shows that exosomes play a critical role in cancer. It has recently been revealed that maternal and umbilical cord serum (UCS)-derived exosomes may enhance endothelial cell proliferation and migration. However, the role of exosomes isolated from the human umbilical cord in cancer development has not been investigated. To explore the potential differences in the composition and function of proteins from umbilical serum exosomes (UEs) and maternal serum exosomes, a proteomic analysis of exosomes was conducted using mass spectrometry and bioinformatics. Moreover, Cell Counting Kit- 8 assays and flow cytometry were used to study the biological effects of UEs on liver cancer cell lines. The present study demonstrated that UCS was enriched with proteins involved in extracellular matrix-receptor interactions, which may be closely related to cell metastasis and proliferation. The findings further indicated that exosomes derived from human umbilical serum could inhibit the viability and induce
\end{abstract}

Correspondence to: Professor Yong Chen, Department of Hepatobiliary Surgery, Xijing Hospital, Fourth Military Medical University, 127 Changle West Road, Xincheng, Xi'an, Shaanxi 710032, P.R. China

E-mail: gdwkcy@163.com

${ }^{*}$ Contributed equally

Key words: exosome, proteomic analysis, human umbilical cord serum, mass spectrometry, liver cancer apoptosis of liver cancer cells. This suggests that UCS-derived exosomes may represent potential leads for the development of biotherapy for liver cancer.

\section{Introduction}

Exosomes are membranous extracellular vesicles 50-100 nm in size, which are involved in cellular communication via the delivery of proteins, lipids and RNA. Tumor derived exosomes could inhibit immune cell proliferation and affect antigen presentation and activation of immune cells (1). In the field of cancer, exosomes from cancer cells may present both immunosuppressive and immunostimulatory properties $(2,3)$. Increasing evidence has shown that exosomes are closely associated with cancer (4) and a previous study demonstrated that exosomes can serve as possible drug delivery vehicles in cancer therapy (5). However, the role of exosomes derived from human umbilical serum in cancer development has not been investigated.

Since pregnancy involves extensive communication among cells and tissues, secreted molecules are thought to participate in cellular signaling, which is important to placentation (6). Extracellular vesicles mediate cell communication. In the process of human pregnancy, elevated levels of circulating maternal exosomes are observed (6). A previous study has reported that exosomes isolated from the human umbilical cord may enhance the angiogenic activities of endothelial cells and endothelial cell proliferation, mostly through microRNA (miRNA) (7). Due to their different cellular origins, exosomes may carry distinct RNAs and protein cargos (8).

A previous study has suggested that exosomes carry bioactive molecules, such as miRNAs, which may participate in the crosstalk between the placenta and maternal tissue (9). It has been demonstrated that differentially expressed maternal serum exosomal miRNA molecules can affect cell growth 
and organ development pathways, and that umbilical serum exosomal miRNA is involved in cellular and embryonic development (10). Thus, different proteins found in exosomes may also be involved in physiological or pathological states (11). Comparing umbilical serum exosomes (UEs) with maternal serum exosomes (MEs) may lead to improved understanding of the differences between the umbilical cord blood and the peripheral blood environments.

A previous study has revealed that human serum may induce hepatoma cell cycle arrest (12). Moreover, to the best of our knowledge, the protein profile of exosomes derived from umbilical cord serum (UCS) using mass spectrometry (MS)/MS has never been described. Thus, the aim of the present study was to explore the potential differences in the composition and function of the proteins found in UEs and MEs, and to investigate the biological effects of UEs on liver cancer cells.

\section{Materials and methods}

Umbilical cord blood extraction. Serum samples were collected at Xijing Hospital (Xi'an, China) from March 2019 to May 2019. The present study was approved by the ethics committee of Xijing Hospital (approval no. 20190105-1) and performed in accordance with their guidelines, with written informed consent obtained for the use of patient tissue and specimens. All experimental protocols were performed in accordance with the relevant Good Laboratory Practice guidelines and regulations of our laboratory. A total of eight healthy pregnant women from Xijing Hospital who were pregnant for the first time and had a natural vaginal delivery were enrolled. The average age of these patients was 29 years old. None of these patients had complications or a history of smoking or alcohol abuse. None of them had taken medication within three months of the study or experienced any infectious diseases. After delivery of the fetus, umbilical cord blood was rapidly collected. The needle was inserted into the umbilical vein and was connected to a negative pressure bag. After insertion of the needle, the umbilical cord blood flowed into the bag through negative pressure. Maternal blood samples were collected from ulnar vein to extract MEs, which were extracted from the same patients as the UEs were extracted from. The samples were centrifuged $1 \mathrm{~h}$ later at $1,000 \mathrm{xg}$ for $30 \mathrm{~min}$ at room temperature, after which the upper layer of clear serum was collected. The samples were then stored at $-130^{\circ} \mathrm{C}$.

Exosome isolation. UEs and MEs were isolated using the ExoQuick ${ }^{\mathrm{TM}}$ Exosome Precipitation Solution (cat.no.EXOQ5A-1; System Biosciences). The procedure of this kit is simple to conduct, and it is efficient at isolating exosomes. Briefly, serum samples were centrifuged at 3,000 x g for $15 \mathrm{~min}$ at $4^{\circ} \mathrm{C}$ to remove clotted materials and cell debris. A volume of $250 \mu \mathrm{l}$ ExoQuick Exosome Precipitation Solution was then added to 1-ml serum supernatants (1:4 ratio of the ExoQuick solution to serum supernatants), and the mixture was refrigerated for $30 \mathrm{~min}$. The mixture was then centrifuged at $1,500 \mathrm{x} \mathrm{g}$ for $30 \mathrm{~min}$ at $4^{\circ} \mathrm{C}$, and the supernatants were discarded. The residual solution was centrifuged at $1,500 \mathrm{x}$ g for $5 \mathrm{~min}$ at $4^{\circ} \mathrm{C}$, and the supernatant was removed. The exosome pellet was resuspended in $500 \mu \mathrm{lBS}$ and stored at $-80^{\circ} \mathrm{C}$.
Trypsin digestion. Dithiothreitol was added to the protein samples to make the final concentration $5 \mathrm{mM}$, and then reduced for $30 \mathrm{~min}$ at $56^{\circ} \mathrm{C}$. Iodoacetamide was then added to the samples to adjust the concentration to $11 \mathrm{mM}$, and the samples were incubated for $15 \mathrm{~min}$ in the dark at room temperature. The protein sample was then diluted by adding $200 \mathrm{mM}$ TEAB (tetraethyl ammonium bromide) to urea concentration $<2 \mathrm{M}$. Finally, trypsin was added at a mass ratio (trypsin:protein) of 1:50 for digestion at $37^{\circ} \mathrm{C}$ overnight. The following day, trypsin was added at a mass ratio of 1:100 for further digestion for $4 \mathrm{~h}$.

TMT (tandem mass tags) labeling. The trypsin-digested peptides were desalted using solid phase extraction cartridges (Strata X C18; Phenomenex) and freeze-dried in a vacuum. The samples were then dissolved with $0.5 \mathrm{M}$ TEAB and the peptides were labeled according to the manufacturer's instructions (TMTsixplex ${ }^{\mathrm{TM}}$ Isobaric Label Reagent; cat. no. 90068; Thermo Fisher Scientific, Inc.).

High-performance liquid chromatography (HPLC) fractionation. Agilent 1260 Infinity Liquid Chromatography System was used at $35^{\circ} \mathrm{C}$. High-pH reverse-phase HPLC was performed to separate the peptides using an Agilent 300 Extend C18 column (Agilent Technologies, Inc.). The column size was ZORBAX Extend-C18 Agilent Analytical 4.6x250-mm 5-Micron 80A. The composition of mobile phase (two solvents) were: Buffer A, $98 \%$ water and $2 \%$ acetonitrile; buffer B, $98 \%$ acetonitrile and $2 \%$ water. The flow rate was used at $1 \mathrm{ml} / \mathrm{min}$. Briefly, the peptides were fractionated into 60 fractions with acetonitrile (8-32\%) at $\mathrm{pH} 9$ for $60 \mathrm{~min}$. The sample quantity was 1,000 $\mu \mathrm{l}$. Pre-loading: Dried sample dissolved in buffer A, and then centrifuged at 18,000 x g for $5 \mathrm{~min}$. Sample combination method: The samples were collected from the 11th min to the 64th min when they flowed out of the liquid phase, and then the first component were combined with the 11th, 29th, 47th and 64th min samples.

$L C-M S / M S$ analysis. The samples were dissolved with $0.1 \%$ aqueous formic acid as the mobile phase using liquid chromatography. The peptides were separated with EASY-nLC 1000 UPLC (Ultra Performance Liquid Chromatography; Thermo Fisher Scientific, Inc.). Mobile phase A was an aqueous solution containing $0.1 \%$ formic acid and $2 \%$ acetonitrile, and mobile phase $\mathrm{B}$ was an aqueous solution containing $0.1 \%$ formic acid and $90 \%$ acetonitrile. The peptides separated by UPLC were then subjected to an Nanospray Ion source. The ion source voltage was set at $2.0 \mathrm{kV}$. An Orbitrap (Thermo Fisher Scientific, Inc.) was used to detect and analyze the peptide parent ions and their secondary fragments. The data acquisition mode used a dependent scanning Data Dependent Acquisition program and the primary scanning range is $350-1,800 \mathrm{~m} / \mathrm{z}$. Finally, the peptides were analyzed by tandem MS/MS in a Q Exactive ${ }^{\mathrm{TM}}$ Plus instrument (Thermo Fisher Scientific, Inc.).

Database search. The MaxQuant (v1.5.2.8, http://www. maxquant.org/) search engine was used to process the mass spectrometry data. The cleavage enzyme was set as trypsin/P, and up to two missing cleavages were allowed. The minimum 
length of the peptide segment was set to seven amino acids, and the maximum modification number of the peptide segment was set to five.

Bioinformatics. The quantitative value of the peptide in each sample was calculated used the ratio of the labeling reporter ion intensities in MS/MS spectra from raw data sets. The median of unique peptides for a given protein was considered as the protein quantitation in each sample. Ratio of protein quantitative value between two samples was considered as the protein expression ratio. In order to calculate the significant P-value of differentially expressed proteins, firstly, perform $\log 2$ transform on the unique peptide quantitative values of the protein in the two samples (making the data conform to the normal distribution), and then the two-sample two-tailed T test method was used to calculate $\mathrm{P}$-value. Proteins with $\mathrm{P}<0.05$ and expression ratio $>1.5$ were regarded as upregulated, while proteins with $\mathrm{P}<0.05$ and expression ratio $<1.5$ were regarded as downregulated.

Gene Ontology (GO) annotation. GO analysis is a kind of bioinformatics tool for characterizing molecular function (MF), cellular component (CC) and biological processes (BP) of genes (13). The protein IDs were determined by MaxQuant (v1.5.2.8; http://www.maxquant.org/) database. Then the protein ID was matched to the UniProt ID based on the UniProt-GOA database and the UniProt ID was used to match the GO ID. Finally, the corresponding information from the UniProt-GOA database was retrieved based on the GO ID. UniProt-GOA database (http://www.ebi.ac.uk/GOA/) and InterProScan v5.14 software (http://www.ebi.ac.uk/interpro/) were used in this section. The enriched proteins based on GO analysis can be divided into three categories: Biological process, cellular component and molecular function. Two-tailed Fisher's exact test was used to determine the GO terms associated with the differentially expressed proteins and $\mathrm{P}<0.05$ was considered significant.

Domain annotation. The InterProScan v5.14 software (http://www.ebi.ac.uk/interpro/) and the InterPro domain database (http://www.ebi.ac.uk/interpro/) were used to analyze the enrichment of the functional domains of the differentially expressed proteins. Two-tailed Fisher's exact test was used to test the enrichment of the differentially expressed proteins and $\mathrm{P}<0.05$ was considered significant.

Kyoto Encyclopedia of Genes and Genomes (KEGG) pathway annotation. The identified proteins were annotated using KAAS (https://www.genome.jp/tools/kaas/) and the annotated proteins were matched to the corresponding pathways using KEGG mapper. Pathway enrichment analysis was based on the KEGG database (https://www.kegg.jp/). Two-tailed Fisher's exact test was used to determine the enriched KEGG pathways associated with the differentially expressed proteins and $\mathrm{P}<0.05$ was considered significant.

Subcellular localization. The cells of eukaryotic organisms are elaborately subdivided into functionally distinct membrane bound compartments (14). Some major constituents of eukaryotic cells are: extracellular space, cytoplasm, nucleus, mitochondria, Golgi apparatus, endoplasmic reticulum (ER), peroxisome, vacuoles, cytoskeleton, nucleoplasm, nucleolus, nuclear matrix and ribosomes. Wolfpsort v0.2 software (https://wolfpsort.hgc.jp/) was used to predict subcellular localization of the identified proteins.

Enrichment-based hierarchical clustering. To identify the functional correlation of the differentially expressed proteins in the comparison groups, hierarchical clustering based on the functional enrichment of the differentially expressed proteins in different groups was used to investigate the potential associations and differences in specific functions (GO terms, KEGG pathways and protein domains) of the proteins. Differentially expressed proteins were categorized into four groups according to their fold change: Q1 (0-0.5), Q2 (0.5-0.67), Q3 (1.5-2) and Q4 ( $>2)$, with $\mathrm{P}<0.05$ in all cases. The functional annotation following enrichment, together with the corresponding enrichment P-value, were first collected. The functional classifications that were enriched in at least one of the clusters (with $\mathrm{P}<0.05)$ were then screened. The filtered P-value data matrix was $-\log _{10}$ transformed. The transformed data matrix was classified by $\mathrm{Z}$ transformation for each functional category. Lastly, the dataset obtained following $\mathrm{Z}$ transformation was analyzed using one-way hierarchical clustering.

Transmission electron microscopy. The exosomes were fixed with $2.5 \%$ glutaraldehyde in PBS for $1 \mathrm{~h}$ at $4^{\circ} \mathrm{C}$. The samples were then centrifuged for $10 \mathrm{~min}$ at $7,000 \mathrm{x} \mathrm{g}$ to remove glutaraldehyde at $4^{\circ} \mathrm{C}$. And the samples were embedded by epoxy resin 618 at $35^{\circ} \mathrm{C}$ for $3 \mathrm{~h}$. Subsequently, the samples were stained with $1 \%$ phosphotungstic acid for $5 \mathrm{~min}$ at room temperature and fixed on copper mesh formvar grids.

Cell culture and Cell Counting Kit-8 (CCK-8) assay. The Huh-7 and MHCC97H hepatocellular carcinoma (HCC) cell lines were purchased from iCell Bioscience, Inc. The liver cancer cell line HepG2 and the HCC cell line Hep3B were purchased from Shanghai GeneChem Co., Ltd. The HepG2 cell line was authenticated by STR profiling. HCC cells and HepG2 liver cancer cell line were cultured in Dulbecco's modified Eagle's medium (HyClone; Cytiva) supplemented with $10 \%$ fetal bovine serum (HyClone; Cytiva) at $37^{\circ} \mathrm{C}$ in $5 \%$ $\mathrm{CO}_{2}$.

Cell viability was measured using the CCK-8 kit (Sigma-Aldrich; Merck KGaA). Cells were seeded in 96-well plates $\left(3 \times 10^{3}\right.$ cells per well) and treated with UEs or MEs at different dilution ratios $(1: 5,1: 10,1: 20,1: 40,1: 80)$ for $24 \mathrm{~h}$ at $37^{\circ} \mathrm{C}$. As a negative control, cells were incubated with medium only. Subsequently, $10 \mu \mathrm{l}$ CCK- 8 reagent was added to the cells for $2 \mathrm{~h}$ at $37^{\circ} \mathrm{C}$. A microplate reader was used to measure the optical density at $450 \mathrm{~nm}$.

Western blot analysis. Cells and exosomes were lysed using RIPA buffer (Thermo Fisher Scientific, Inc.) with proteinase and phosphatase inhibitors (Roche Diagnostics). Total protein was quantified using a Pierce BCA Protein Assay kit (Thermo Fisher Scientific, Inc.). The mass of protein loaded per lane was $50 \mu \mathrm{g}$. Total protein was separated by SDS-PAGE on $10 \%$ gels, then transferred to polyvinylidene fluoride membranes (EMD Millipore). The membranes 


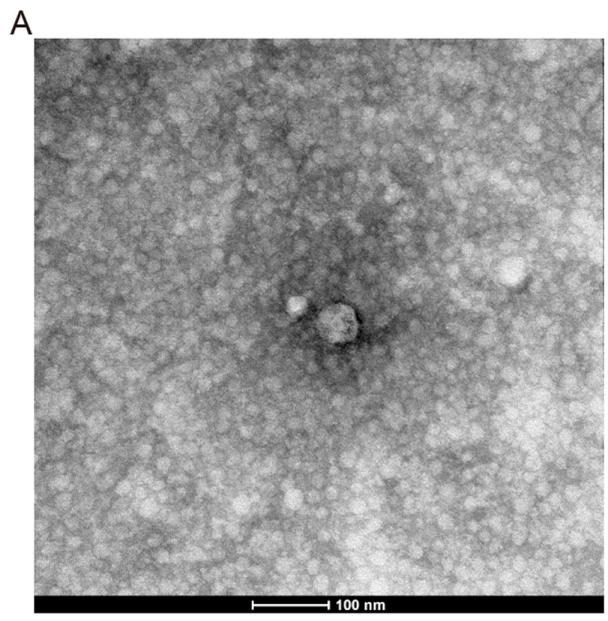

ME
$\mathrm{B}$

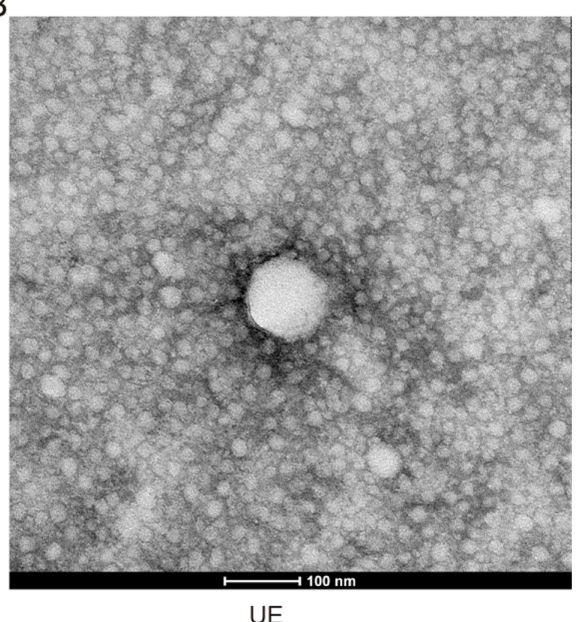

C

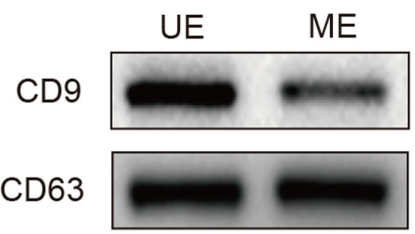

Figure 1. Transmission electron microscopy and western blot analysis of exosomes isolated from serum samples. (A) MEs and (B) UEs were examined using transmission electron microscopy. (C) Western blot analysis of CD9 and CD36 expression in MEs and UEs. ME, maternal serum exosome; UE, umbilical serum exosome.

were blocked with $5 \%$ non-fat milk at room temperature for $1 \mathrm{~h}$ and incubated with primary antibodies overnight at $4^{\circ} \mathrm{C}$. The following antibodies were used: i) Anti-CD63 (cat. no. ab134045; Abcam); ii) anti-CD9 (cat. no. ab236630; Abcam); iii) anti-caspase-3 (cat. no. \#9662; Cell Signaling Technology, Inc.); iv) anti-cleaved-caspase-3 (cat. no. \#9661; Cell Signaling Technology, Inc.); and v) and anti-GAPDH antibody (cat. no. \#5174; Cell Signaling Technology, Inc.). All primary antibodies were monoclonal and used at 1:1,000 dilution. The membranes were incubated with secondary antibodies for $1 \mathrm{~h}$ on the second day at room temperature. The peroxidase-conjugated anti-rabbit secondary antibodies (cat. no. A6154; Sigma-Aldrich; Merck KGaA) was used in 1:5,000 dilution. Membranes were finally treated with enhanced chemiluminescence reagent (Thermo Fisher Scientific, Inc.) and exposed to chemiluminescence imaging systems (version 5.11 Bio-Rad Laboratories).

Flow cytometry. After treatment with UEs (dilution ratio, 1:5) or MEs (dilution ratios, 1:5) for $24 \mathrm{~h}$ at $37^{\circ} \mathrm{C}$, cells were digested and resuspended in PBS at $1 \times 10^{6} / \mathrm{ml}$. The samples were then incubated with $10 \mu$ l Annexin V-FITC (Sigma-Aldrich; Merck KGaA) and $10 \mu \mathrm{l}$ propidium iodide (Sigma-Aldrich; Merck KGaA) for 10-15 $\mathrm{min}$ at room temperature in the dark. Finally, flow cytometry (BD FACSCalibur; BD Biosciences) was used to analyze the cells. And the data was analyzed by DIVA version 11.0 software (BD Biosciences).

Statistical analysis. Statistical analysis was performed using SPSS 22.0 (IBM Corp.). A paired Student's t-test was used to compare the differences between two groups. Comparisons among three groups were analyzed using one-way ANOVA followed by Bonferroni correction. $\mathrm{P}<0.05$ was considered to indicate a statistically significant difference.

\section{Results}

Exosome isolation from serum samples. Exosomes were isolated from serum samples and identified using transmission electron microscopy and western blot analysis (15). The isolated extracellular vesicles were found to have a diameter of 50-100 nm (Fig. 1A and B). CD9 and CD63 were detected in the protein samples extracted from the exosomes (Fig. 1C). The protein concentration in the exosome samples was $1.5 \pm 0.13 \mu \mathrm{g} / \mu \mathrm{l}$ in the UE group and $1.1 \pm 0.10 \mu \mathrm{g} / \mu \mathrm{l}$ in the ME group.

Comparison of the protein profiles between UEs and MEs. The protein profiles of the exosomes from the serum samples were then obtained, and 471 proteins were identified. Of these, 193 proteins were differentially expressed between the two groups, including 128 upregulated proteins and 65 downregulated proteins in UEs relative to MEs (Fig. 2A). Representative mass spectra data of some of the differentially expressed proteins are provided in Table SI. Representative mass spectra images of the identified proteins are provided in Fig. S1. Wolfpsort was then used to predict subcellular localization. The upregulated proteins were predicted to be from the extracellular region (48\%), followed by the cytoplasm (26\%), plasma membrane (7\%), nucleus (5\%), mitochondria (5\%), endoplasmic reticulum $(4 \%)$, peroxisome (2\%), cytoplasm and nucleus $(1 \%)$, and cytoskeleton (2\%) (Fig. 2B and C). The downregulated proteins were from the extracellular region $(77 \%)$, followed by the 
A

Differentially expressed proteins

\begin{tabular}{lcc}
\hline Group & fold change $>1.5$ & fold change $<0.67$ \\
\hline UEs/MEs & 128 & 65 \\
\hline
\end{tabular}

B

Predicted subcellular location

of upregulated proteins

\begin{tabular}{cc}
\hline Subcellular location & No. of proteins \\
\hline Extracellular & 61 \\
Cytoplasm & 33 \\
Plasma membrane & 9 \\
Nucleus & 7 \\
Mitochondria & 6 \\
Endoplasmic reticulum & 5 \\
Peroxisome & 3 \\
Cytoplasm and nucleus & 2 \\
Cytoskeleton & 2 \\
\hline Total & 128 \\
\hline
\end{tabular}

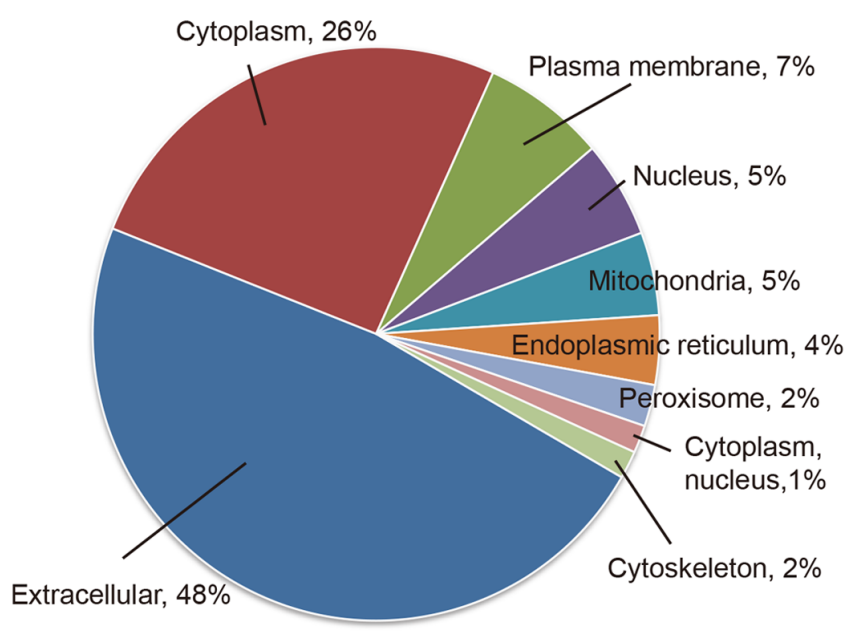

D

Predicted subcellular Location of downregulated proteins

\begin{tabular}{cc}
\hline Subcellular location & No. of proteins \\
\hline Extracellular & 50 \\
Endoplasmic reticulum & 4 \\
Plasma membrane & 3 \\
Nucleus & 3 \\
Mitochondria & 3 \\
Cytoplasm & 2 \\
\hline Total & 65 \\
\hline
\end{tabular}

E

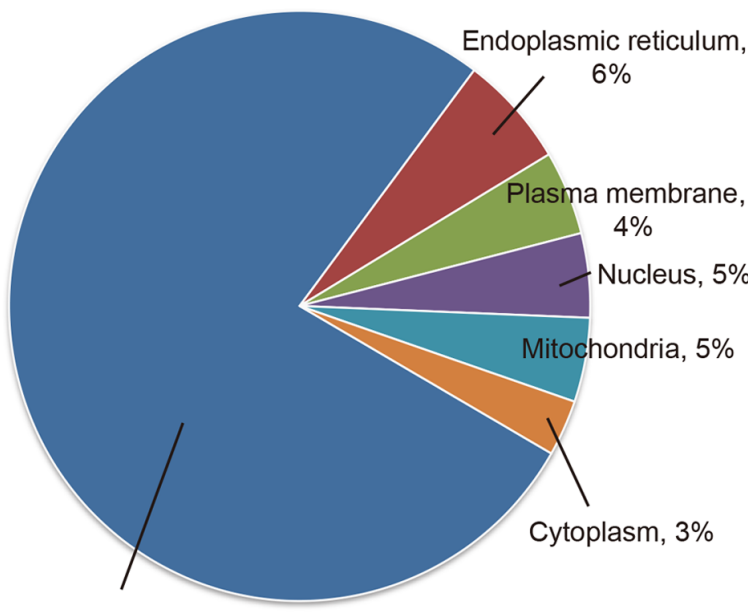

Extracellular, $77 \%$

Figure 2. Comparison of the protein profiles of UEs and MEs. (A) Differentially expressed proteins were identified between the groups, including 128 upregulated proteins (fold-change $>1.5 ; \mathrm{P}<0.05$ ) and 65 downregulated proteins (fold-change $<0.67 ; \mathrm{P}<0.05$ ) in UEs. (B) Predicted subcellular location of the upregulated proteins and number of corresponding proteins. (C) Percentage of upregulated proteins with their predicted subcellular localization. (D) Predicted subcellular location of the downregulated proteins and number of corresponding proteins. (E) Percentage of downregulated proteins with their predicted subcellular localization. ME, maternal serum exosome; UE, umbilical serum exosome.

endoplasmic reticulum (6\%), nucleus $(5 \%)$, mitochondria $(5 \%)$, plasma membrane (4\%) and cytoplasm (3\%) (Fig. 2D and E).

Functional enrichment analysis of differentially expressed proteins. The differentially expressed proteins identified in the present study were examined by GO analysis and categorized as cellular component, molecular function and biological process terms. The upregulated proteins derived from UEs were mostly enriched in the 'extracellular matrix' cellular component, 'cell adhesion molecule binding' molecular function, and 'cell adhesion' and 'biological adhesion' biological processes (Fig. 3A). The downregulated proteins were mainly enriched in 'plasma lipoprotein particles', 'protein-lipid complex' and 'lipoprotein particle' in the cellular component category, as well as the 'enzyme inhibitor activity' molecular function and the 'acute inflammatory response' biological process (Fig. 3B). Thus, the differentially expressed proteins in the UCS may participate in cell adhesion and cell junctions.

KEGG pathway enrichment of differentially expressed proteins. KEGG pathway analysis predicted that the enriched proteins derived from UCS were associated with 
A

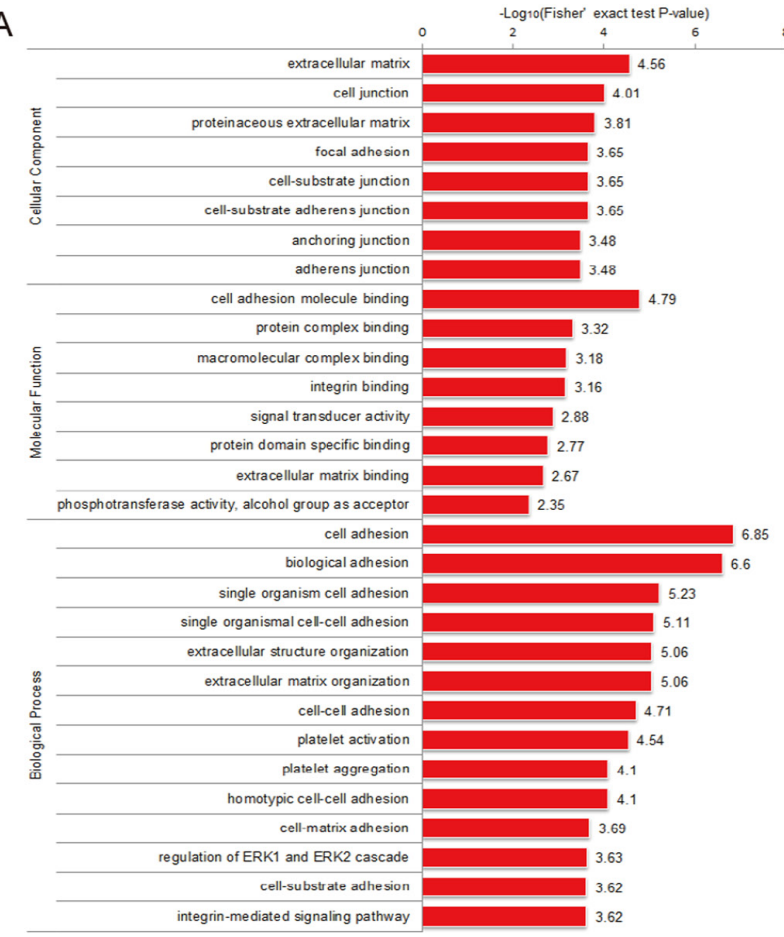

$\therefore B$

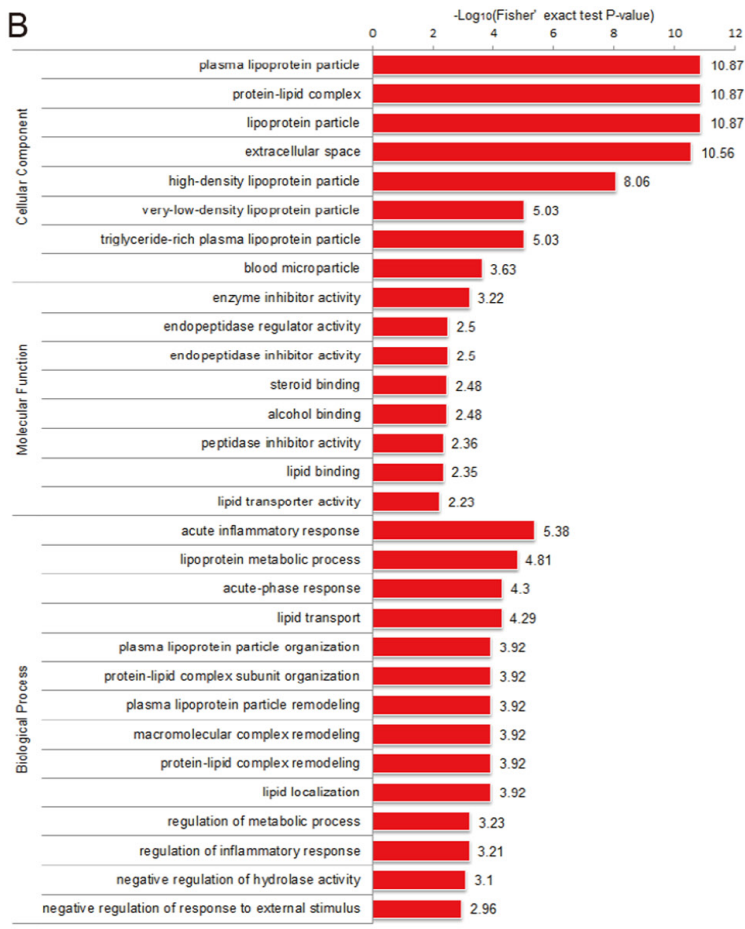

Figure 3. GO enrichment analysis of differentially expressed proteins. (A) Upregulated and (B) downregulated proteins were examined using GO analysis and categorized as cellular components, molecular functions and biological processes. GO, Gene Ontology.

the 'ECM-receptor interaction' pathways (Fig. 4A), including the 'ECM-integrin' and the 'ECM-proteoglycan' cascades. According to the functional enrichment analysis based on the KEGG pathway, 'focal adhesion' and 'ECM-receptor interaction' were the most enriched pathways (Fig. 4B).

Protein domain enrichment of differentially expressed proteins. Protein domains are elementary units of protein structure and evolution, which mediate most ( $75 \%)$ protein interactions $(16,17)$. The length of the domain is usually between 25 amino acids and 500 amino acids (18-20). The enriched domain of the upregulated proteins was mainly 'galactose-binding domain-like', whereas the downregulated proteins were enriched in the 'serine proteases, trypsin domain' and 'peptidase S1, PA clan' protein domains (Fig. 4C and D).

Hierarchical clustering of the functional annotation of differentially expressed proteins. Hierarchical clustering based on GO analysis in the cellular component category showed that the differentially expressed proteins of Q1 were mainly involved in, for example, 'very-low-density lipoprotein particle', 'chylomicron' and 'high-density lipoprotein particle' (Fig. S2). Q2 were mainly involved in 'membrane attack complex' and 'pore complex' in the cellular component category. Q3 were mainly involved in 'blood microparticle' in the cellular component category. While Q4 were mainly involved in 'proteinaceous extracellular matrix', 'extracellular matrix' and 'cell junction' in the cellular component category (Fig. S2). Hierarchical clustering based on GO analysis in the biological process category showed that the differentially expressed proteins of Q1 were mainly involved in 'acute inflammatory response' and 'acute-phase response' (Fig. S3). Q2 were mainly involved in 'negative regulation of response to external stimulus' and 'regulation of proteolysis' in the biological process category. Q3 were mainly involved in 'positive regulation of immune response' and 'protein activation cascade' in the biological process category. Q4 were mainly involved in 'positive regulation of response to external stimulus' in the biological process category (Fig. S3). Hierarchical clustering based on GO analysis in the molecular function category showed that the differentially expressed proteins of Q1 were mainly involved in 'steroid binding' (Fig. S4). Q2 were mainly involved in 'peptidase inhibitor activity' and 'endopeptidase regulator activity' in the molecular function category. Q3 were mainly involved in 'endopeptidase activity' and 'hydrolase activity' in the molecular function category. Q4 were mainly involved in 'protein complex binding' and 'integrin binding' in the molecular function category (Fig. S4).

Hierarchical clustering based on protein domain analysis revealed that UCS proteins of Q1 were mostly enriched with 'serine proteases, trypsin domain' and 'peptidase S1, PA clan', while Q2-Q4 showed a lower degree of enrichment with 'serine proteases, trypsin domain' and 'peptidase S1, PA clan' (Fig. 5B).

Hierarchical clustering based on KEGG pathway analysis showed that UCS proteins of Q4 were mainly involved in 'ECM-receptor interaction', 'focal adhesion', 'platelet activation', 'toxoplasmosis', 'regulation of actin cytoskeleton' and 'PI3K-Akt signaling pathway', while Q1-Q3 showed a lower degree of enrichment in these terms (Fig. 5C).

Effect of UEs on liver cancer cells. To investigate the effects of exosomes derived from human UCS on liver cancer cells, a CCK-8 assay was conducted to detect cell viability following treatment with UE. As shown in Fig. 6A, Hep3B cells were cultured with exosomes at different dilution ratios. Cell viability was significantly reduced in Hep3B cells treated 
A
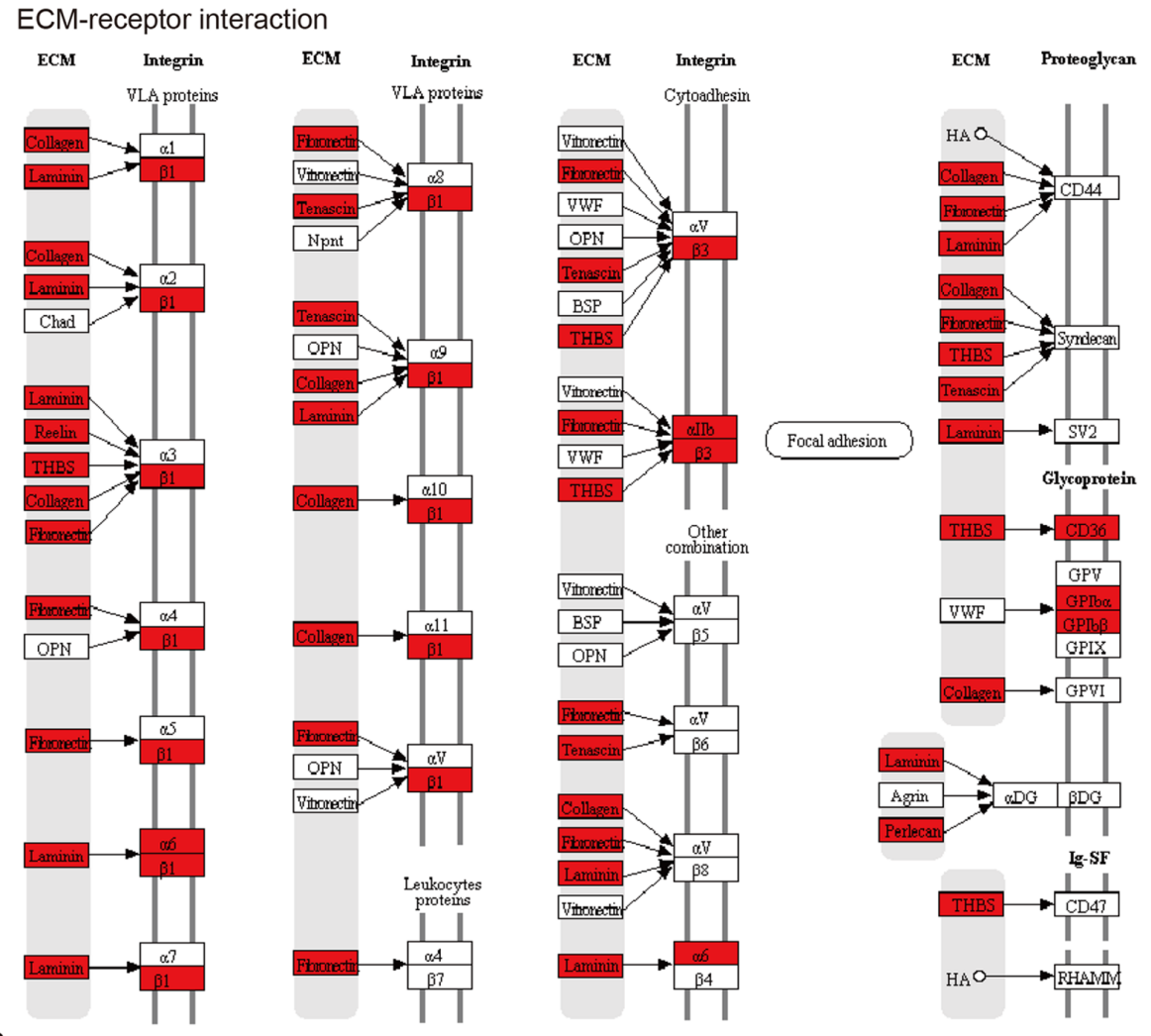

B

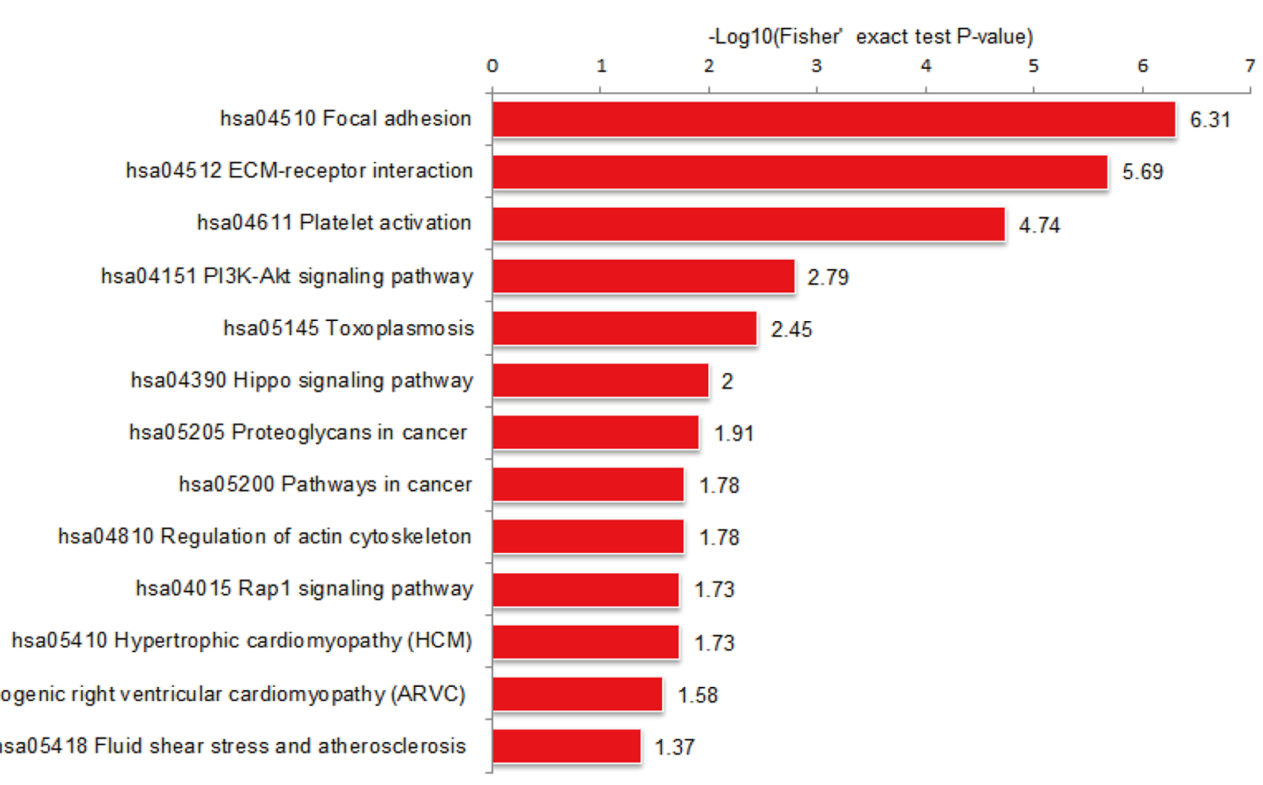

C

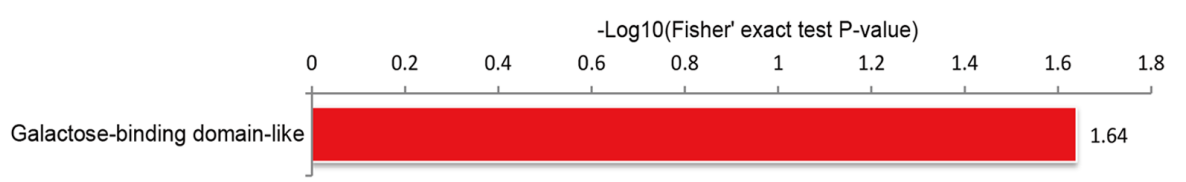

D

\begin{tabular}{|c|c|c|c|c|c|}
\hline & & & t $P-v$ & & \\
\hline 0 & 0.5 & 1 & 1.5 & 2 & 2.5 \\
\hline Serine proteases, trypsin domai & & & & & 2.16 \\
\hline Peptidase S1, PA clan & & & & & 2.16 \\
\hline
\end{tabular}

Figure 4. KEGG pathway and protein domain enrichment of differentially expressed proteins. (A) The illustration from the KEGG database showing that the enriched proteins derived from umbilical cord serum were involved in ECM-receptor interactions. (B) KEGG pathway enrichment analysis showing that 'focal adhesion' and 'ECM-receptor interaction' were the most enriched pathways. (C) Protein domain enrichment analysis of high-abundance proteins mainly involved the 'galactose-binding domain-like' domain. (D) Protein domain enrichment analysis of low-abundance proteins mainly involved the 'serine proteases, trypsin domain' and 'peptidase S1, PA clan' domains. KEGG, Kyoto Encyclopedia of Genes and Genomes; ECM, extracellular matrix. 
A

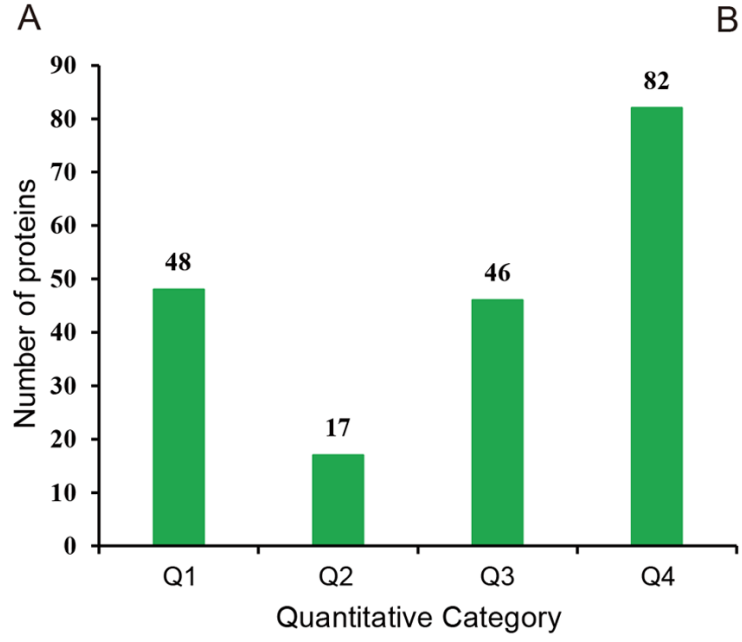

C

KEGG pathway

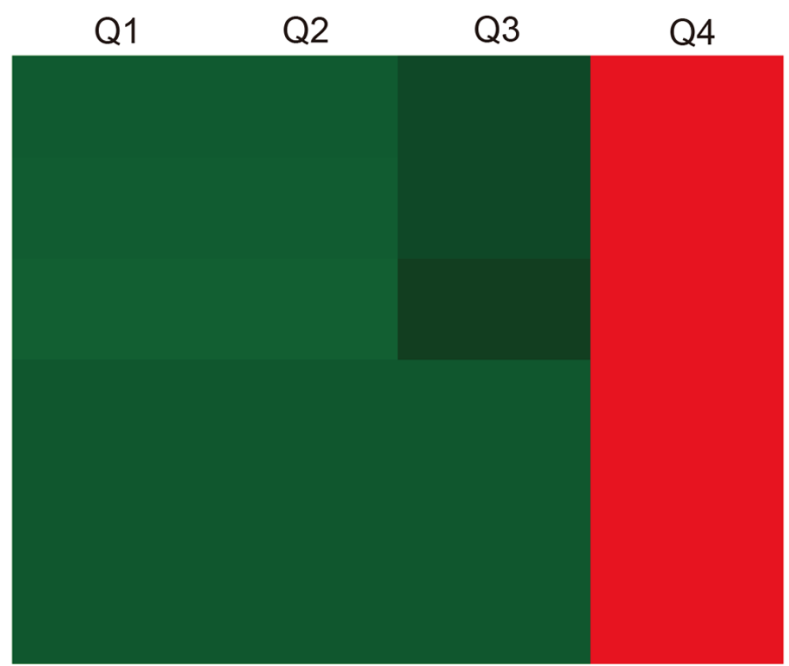

Protein domain

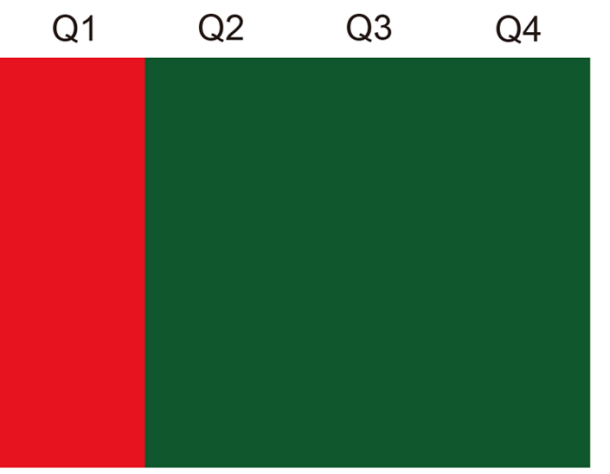

Serine proteases, trypsin domain

Peptidase S1, PA clan

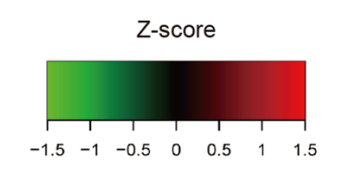

hsa04512 ECM-receptor interaction-Homo sapiens(human)

hsa04510 Focal adhesion-Homo sapiens(human)

hsa04611 Platelet activation-Homo sapiens(human)

hsa05145 Toxoplasmosis-Homo sapiens(human)

hsa04810 Regulation of actin cytoskeleton-Homo sapiens(human)

hsa04151 PI3K-Akt signaling pathway-Homo sapiens(human)

Figure 5. Hierarchical clustering analysis of differentially expressed proteins. (A) Differentially expressed proteins were categorized into four groups according to their fold change: Q1 (0-0.5), Q2 (0.5-0.67), Q3 (1.5-2) and Q4 (>2), with P<0.05 in all cases. (B) A heatmap was generated from hierarchical clustering results of the protein domain analysis. UCS proteins were mostly enriched with 'serine proteases, trypsin domain' and 'peptidase S1, PA clan' domains. (C) A heatmap was generated from hierarchical clustering results of KEGG pathway analysis. UCS proteins were mostly involved in 'ECM-receptor interaction' and 'focal adhesion'. Green represents a lower degree of domain enrichment, and red indicates a higher degree of enrichment relatively. KEGG, Kyoto Encyclopedia of Genes and Genomes; UCS, umbilical cord serum.

with UEs compared with cells treated with MEs or with the negative control group. The inhibitory effect of UEs started at a dilution ratio of 1:20. Subsequently, cell viability was detected in three liver cancer cell lines following treatment with UEs at different dilution ratios. The results showed that exosomes from human UCS could reduce the viability of liver cancer cells compared with the normal human liver cell line (QSG-7701) (Fig. 6B). To further validate this result, cell viability was detected in Hep3B cells treated with MEs and UEs ( $n=8$ in each group). In 6 out 8 cases, UEs significantly suppressed cell viability compared with MEs (Fig. 6C). These results indicated that exosomes derived from human UCS could suppress liver cancer cell viability.

UEs may induce apoptosis of HCC cells. To investigate the effect of UEs on cell apoptosis, flow cytometry was used to determine apoptotic cell death in HCC cells. UE treatment significantly increased the percentage of early apoptosis (lower-right quadrant) and late apoptosis (upper-right quadrant) (Figs. 6D and S5). To further demonstrate that UEs can induce apoptosis of HCC cells, the protein expression levels of caspase- 3 and cleaved-caspase- 3 were determined. The results suggested that the expression of cleaved caspase-3 was significantly higher in the UE group than in the ME and the negative control groups (Fig. 6E and F). This indicated that exosomes isolated from UCS could induce apoptosis of HCC cells.

\section{Discussion}

Exosomes are subcellular vesicles consisting mainly of phospholipids, proteins, cholesterol, ceramide and sphingolipids. The morphological and biochemical properties of exosomes vary according to their cellular origin $(21,22)$. Exosomes are extracted and isolated mainly by ultrafast centrifugation, 
A

Нер3В

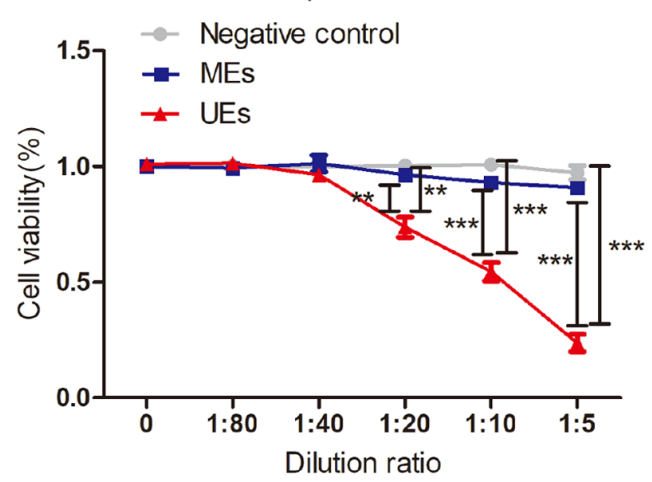

B

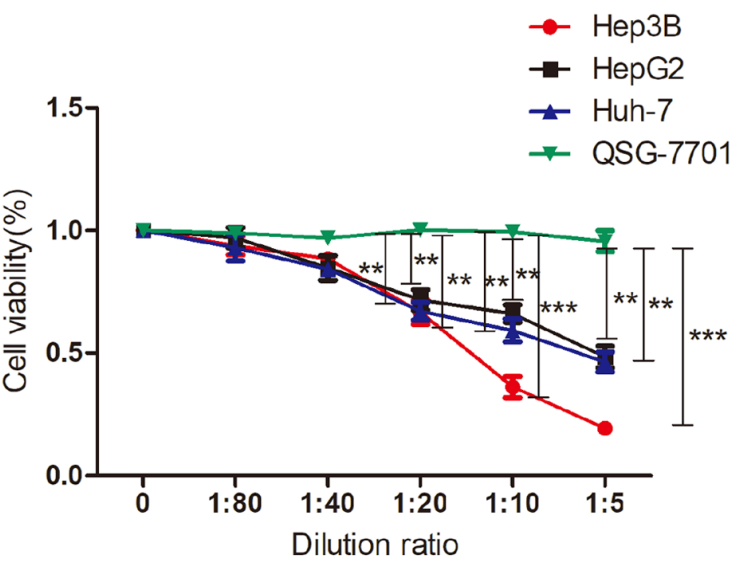

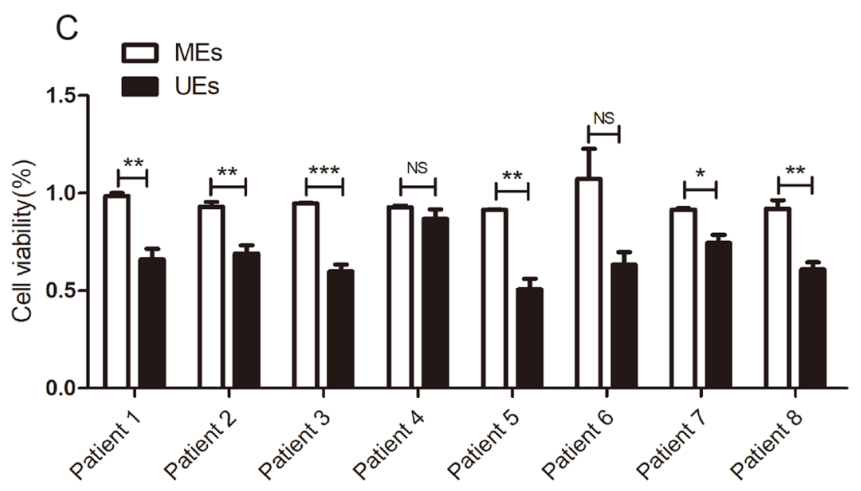
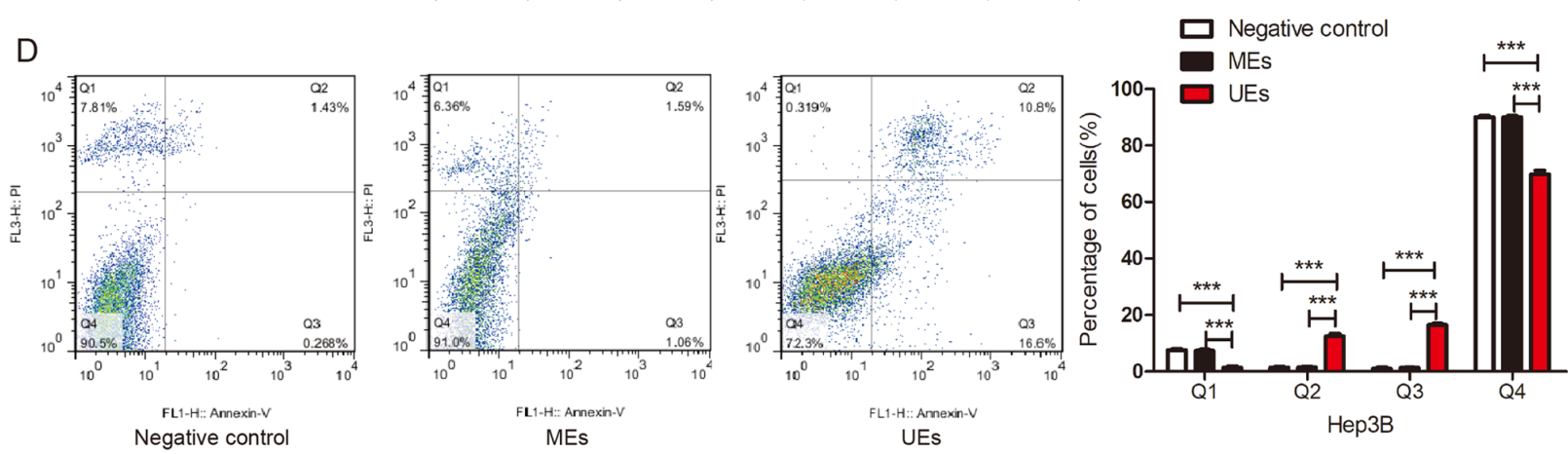

E

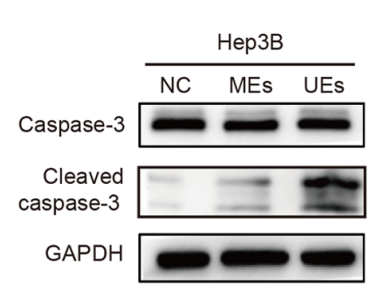

$\mathrm{F}$

Hep3B

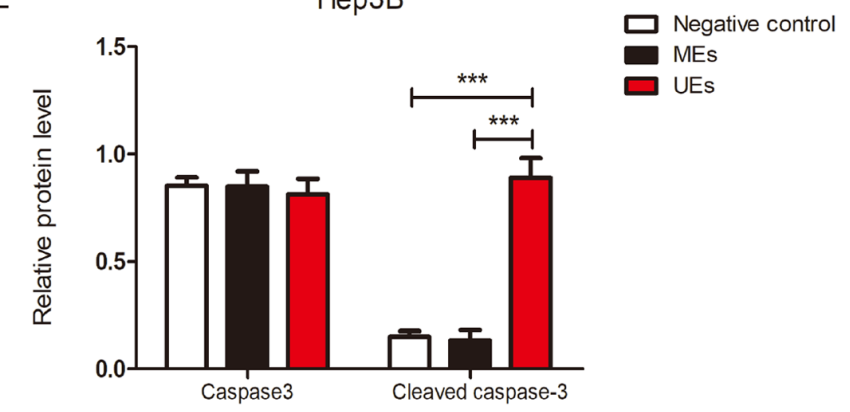

Figure 6. Effects of exosomes derived from human umbilical serum on liver cancer cells. (A) Viability of Hep3B cells following culture with exosomes from different groups (negative control, ME and UE groups) and at different dilution ratios. (B) Viability in HCC cells and HepG2 cell line and normal human liver cell line following treatment with UEs at different dilution ratios. (HCC cells vs. QSG-7701 or HepG2 vs. QSG-7701) (C) Viability in Hep3B cells treated with MEs and UEs isolated from eight patients and corresponding umbilical cord samples. (D) Flow cytometry was used to determine apoptotic cell death of Hep3B cells treated with exosomes in different groups (negative control, ME and UE groups). (E) Western blot analysis was conducted to detect the protein expression levels of caspase-3 and cleaved-caspase-3 in different groups (negative control, ME and UE groups). (F) Semi-quantification of the western blot experiments. The data are presented as the mean \pm SEM. ${ }^{*} \mathrm{P}<0.05,{ }^{* *} \mathrm{P}<0.01,{ }^{* * * *} \mathrm{P}<0.001$. ME, maternal serum exosome; UE, umbilical serum exosome; PI, propidium iodide.

immunomagnetic bead separation, precipitation or filtration, among which ultrafast centrifugation is considered the most common method to separate exosomes at present, and is also the gold standard for exosome extraction and identification $(23,24)$.
Exosomes were extracted from UCS and maternal serum samples. Transmission electron microscopy and western blotting were then used to identify the exosomes. Nearly all of the isolated extracellular vesicles had a diameter of 
50-100 $\mathrm{nm}$ according to the transmission electron microscopy results (25). In addition, CD9 and CD63 were detected in the protein samples extracted from the exosomes. CD9 and CD63 are molecular markers of exosomes (26). These results confirmed that exosomes were successfully isolated from UCS and maternal serum samples.

Exosomes can be found in several types of body fluids, such as blood, saliva, urine, cerebrospinal fluid and milk $(27,28)$. Compared with the relatively simple embryonic environment of umbilical cord blood, the physiological or pathological environment of peripheral blood is more complex (29). Therefore, comparing UEs with MEs may help us better understand the differences between umbilical cord blood and the peripheral blood environment. A recent study revealed that UEs and MEs may enhance endothelial cell proliferation and migration (30); however, the role of exosomes isolated from the human umbilical cord in cancer development has not been investigated. To explore the potential differences in the composition and function of proteins from UEs and MEs, a proteomic analysis of exosomes was conducted by mass spectrometry and bioinformatics analysis. To study the biological effects of UEs on liver cancer cells and to explore their potential value as a new approach for liver cancer biotherapy, MEs were used as a control.

According to the results of the proteomic analysis of exosomes by mass spectrometry and bioinformatics analysis, UEs were enriched with proteins that were involved in 'ECM-receptor interaction'. According to the functional enrichment analysis based on KEGG pathways, these differentially expressed proteins were associated with the integrin family. Integrin expression levels have been revealed to be closely associated with tumor development. The integrin family may be closely related to cell metastasis and proliferation $(31,32)$. Integrin $\beta 1$ has been reported to be mainly involved in adhesion between cells and the extracellular matrix (33), and its activity may affect the distribution and differentiation of stem cells (34). Moreover, integrin $\beta 1$ has been shown to be significantly upregulated in liver cancer and may serve as bidirectional transducers of extracellular and intracellular signals in the processes of cell adhesion (35). Therefore, a large number of differentially expressed proteins in exosomes derived from UCS may be related to the differentiation of a variety of stem cells, and may also be involved in cell adhesion, migration, apoptosis and other biological processes.

To investigate the effects of UEs on liver cancer cells, CCK-8 assays were conducted to detect cell viability in different groups. Exosomes derived from human UCS could suppress liver cancer cell viability, especially Hep3B cells. However, exosomes from UCS had no significant effect on the migration of these cells (data not shown). Flow cytometry was used to determine apoptotic cell death of HCC cells to investigate the effect of UEs on cell apoptosis. The results indicated that UEs could induce apoptosis of HCC cells. Exosomes often contain signaling molecules. In the present bioinformatics analysis, KEGG pathways associated with signaling were identified, such as 'PI3K-AKT signaling' pathway. However, whether they are related to apoptosis induced by exosomes is unknown.

In conclusion, exosomes were isolated from UCS and maternal serum samples, and to the best of our knowledge, the present study was the first to conduct a proteomic analysis of these samples using mass spectrometry. The present study demonstrated that UCS was enriched with proteins involved in ECM-receptor interactions. These differentially expressed proteins may act on the integrin family, which could be related to cell metastasis and proliferation. These findings indicated that exosomes derived from human UCS can suppress the viability of liver cancer cells and induce apoptosis of HCC cells. Further studies are needed to elucidate the mechanism through which UEs suppress liver cancer cell viability and induce apoptosis of HCC cells.

\section{Acknowledgements}

The authors thank Dr Mingzuo Jiang (Department of Gastroenterology and Hepatology, Jinling Hospital, Medical School of Nanjing University) for their technical help.

\section{Funding}

This study was supported by grants from The National Key R\&D Program of China (grant no. 2017YFC1308600), The Key Research and Development Program of Shaanxi Province (grant no. 2017ZDXM-SF-024) and The National Natural Science Foundation of China (grant nos. 81670563 and 31900566).

\section{Availability of data and materials}

The data supporting the findings of the article is available in the PRIDE database (https://www.ebi.ac.uk/pride/) under accession no. PXD025079.

\section{Authors' contributions}

DZ designed the study. DZ, CF and RY performed the experiments. WL and YC contributed to data analysis. MJ, YC and $\mathrm{XL}$ performed data interpretation. YC contributed to the critical revision of the manuscript. DZ wrote the manuscript. DZ and CF confirm the authenticity of all the raw data. All authors approved the final version of the manuscript.

\section{Ethics approval and consent to participate}

This study was approved by The Ethics Committee of Xijing Hospital. Written informed consent was obtained from the patients.

\section{Patient consent for publication}

Not applicable.

\section{Competing interests}

The authors declare that they have no competing interests.

\section{References}

1. Olejarz W, Dominiak A, Żołnierzak A, Kubiak-Tomaszewska G and Lorenc T: Tumor-Derived exosomes in immunosuppression and immunotherapy. J Immunol Res 2020: 6272498, 2020.

2. Chen G, Huang AC, Zhang W, Zhang G, Wu M, Xu W, Yu Z, Yang J, Wang B, Sun H, et al: Exosomal PD-L1 contributes to immunosuppression and is associated with anti-PD-1 response. Nature 560: 382-386, 2018 
3. Morishita M, Takahashi Y, Matsumoto A, Nishikawa M and Takakura Y: Exosome-based tumor antigens-adjuvant co-delivery utilizing genetically engineered tumor cell-derived exosomes with immunostimulatory CpG DNA. Biomaterials 111: $55-65,2016$.

4. Yu DD, Wu Y, Shen HY, Lv MM, Chen WX, Zhang XH, Zhong SL, Tang JH and Zhao JH: Exosomes in development, metastasis and drug resistance of breast cancer. Cancer Sci 106 : 959-964, 2015

5. Kibria G, Ramos EK, Wan Y, Gius DR and Liu H: Exosomes as a drug delivery system in cancer therapy: Potential and challenges. Mol Pharm 15: 3625-3633, 2018.

6. Mincheva-Nilsson L and Baranov V: Placenta-derived exosomes and syncytiotrophoblast microparticles and their role in human reproduction: Immune modulation for pregnancy success. Am J Reprod Immunol 72: 440-457, 2014.

7. Hu Y, Rao SS, Wang ZX, Cao J, Tan YJ, Luo J, Li HM, Zhang WS, Chen CY and Xie H: Exosomes from human umbilical cord blood accelerate cutaneous wound healing through miR-21-3p-mediated promotion of angiogenesis and fibroblast function. Theranostics 8: 169-184, 2018.

8. La Marca V and Fierabracci A: Insights into the diagnostic potential of extracellular vesicles and their miRNA signature from liquid biopsy as early biomarkers of diabetic micro/macrovascular complications. Int J Mol Sci 18: 1974, 2017.

9. Salomon C and Rice GE: Role of exosomes in placental homeostasis and pregnancy disorders. Prog Mol Biol Transl Sci 145 163-179, 2017.

10. Cleys ER, Halleran JL, McWhorter E, Hergenreder J, Enriquez VA, da Silveira JC, Bruemmer JE, Winger QA and Bouma GJ Identification of microRNAs in exosomes isolated from serum and umbilical cord blood, as well as placentomes of gestational day 90 pregnant sheep. Mol Reprod Dev 81: 983-993, 2014.

11. Raposo G and Stoorvogel W: Extracellular vesicles: Exosomes, microvesicles, and friends. J Cell Biol 200: 373-383, 2013.

12. Steenbergen RH, Joyce MA, Thomas BS, Jones D, Law J, Russell R, Houghton M and Tyrrell DL: Human serum leads to differentiation of human hepatoma cells, restoration of very-low-density lipoprotein secretion, and a 1000 -fold increase in HCV Japanese fulminant hepatitis type 1 titers. Hepatology 58 : 1907-1917, 2013.

13. Piao C, Zhang Q, Jin D, Wang L, Tang C, Zhang N, Lian F and Tong X: A study on the mechanism of milkvetch root in the treatment of diabetic nephropathy based on network pharmacology. Evid Based Complement Alternat Med 2020: 6754761, 2020

14. Xie S, Du Y, Zhang Y, Wang Z, Zhang D, He L, Qiu L, Jiang J and Tan W: Aptamer-based optical manipulation of protein subcellular localization in cells. Nat Commun 11: 1347, 2020.

15. Andre Mdo R, Pedro A and Lyden D: Cancer exosomes as mediators of drug resistance. Methods Mol Biol 1395: 229-239, 2016.

16. Przytycka T, Davis G, Song N and Durand D: Graph theoretical insights into evolution of multidomain proteins. J Comput Biol 13: 351-363, 2006

17. Peterson TA, Nehrt NL, Park D and Kann MG: Incorporating molecular and functional context into the analysis and prioritization of human variants associated with cancer. J Am Med Inform Assoc 19: 275-283, 2012.

18. Dawson N, Sillitoe I, Marsden RL and Orengo CA: The classification of protein domains. Methods Mol Biol 1525: 137-164, 2017.

19. Mehrotra P, Ami VKG and Srinivasan N: Clustering of multi-domain protein sequences. Proteins 86: 759-776, 2018.

20. Sawyer N, Watkins AM and Arora PS: Protein domain mimics as modulators of protein-protein interactions. Acc Chem Res 50: 1313-1322, 2017.
21. Corrado C, Raimondo S, Chiesi A, Ciccia F, De Leo G and Alessandro R: Exosomes as intercellular signaling organelles involved in health and disease: Basic science and clinical applications. Int J Mol Sci 14: 5338-5366, 2013.

22. Barile L and Vassalli G: Exosomes: Therapy delivery tools and biomarkers of diseases. Pharmacol Ther 174: 63-78, 2017.

23. Wu X, Showiheen SAA, Sun AR, Crawford R, Xiao Y, Mao X and Prasadam I: Exosomes extraction and identification. Methods Mol Biol 2054: 81-91, 2019.

24. Tang YT, Huang YY, Zheng L, Qin SH, Xu XP, An TX, Xu Y, Wu YS, Hu XM, Ping BH and Wang Q: Comparison of isolation methods of exosomes and exosomal RNA from cell culture medium and serum. Int J Mol Med 40: 834-844, 2017.

25. Cizmar P and Yuana Y: Detection and characterization of extracellular vesicles by transmission and cryo-transmission electron microscopy. Methods Mol Biol 1660: 221-232, 2017.

26. Khushman M, Bhardwaj A, Patel GK, Laurini JA, Roveda K, Tan MC, Patton MC, Singh S, Taylor W and Singh AP: Exosomal markers (CD63 and CD9) expression pattern using immunohistochemistry in resected malignant and nonmalignant pancreatic specimens. Pancreas 46: 782-788, 2017.

27. Flaumenhaft R: Formation and fate of platelet microparticles. Blood Cells Mol Dis 36: 182-187, 2006.

28. Zmigrodzka M, Guzera M, Miskiewicz A, Jagielski D and Winnicka A: The biology of extracellular vesicles with focus on platelet microparticles and their role in cancer development and progression. Tumour Biol 37: 14391-14401, 2016.

29. Sadovsky Y, Mouillet JF, Ouyang Y, Bayer A and Coyne CB: The function of TrophomiRs and other MicroRNAs in the human placenta. Cold Spring Harb Perspect Med 5: a023036, 2015.

30. Jia L, Zhou X, Huang X, Xu X, Jia Y, Wu Y, Yao J, Wu Y and Wang K: Maternal and umbilical cord serum-derived exosomes enhance endothelial cell proliferation and migration. FASEB J 32: 4534-4543, 2018.

31. Ata R and Antonescu CN: Integrins and cell metabolism: An intimate relationship impacting cancer. Int J Mol Sci 18: 189, 2017.

32. Yin HL, Wu CC, Lin CH, Chai CY, Hou MF, Chang SJ, Tsai HP, Hung WC, Pan MR and Luo CW: $\beta 1$ integrin as a prognostic and predictive marker in triple-negative breast cancer. Int $\mathrm{J}$ Mol Sci 17: 1432, 2016

33. Begum A, Ewachiw T, Jung C, Huang A, Norberg KJ, Marchionni L, McMillan R, Penchev V, Rajeshkumar NV, Maitra A, et al: The extracellular matrix and focal adhesion kinase signaling regulate cancer stem cell function in pancreatic ductal adenocarcinoma. PLoS One 12: e0180181, 2017.

34. Quisenberry CR, Nazempour A, Van Wie BJ and Abu-Lail NI: Evaluation of $\beta 1$-integrin expression on chondrogenically differentiating human adipose-derived stem cells using atomic force microscopy. Biointerphases 11: 021005, 2016.

35. Li Y, Ren Z, Wang Y, Dang YZ, Meng BX, Wang GD, Zhang J, $\mathrm{Wu}$ J and Wen N: ADAM17 promotes cell migration and invasion through the integrin $\beta 1$ pathway in hepatocellular carcinoma. Exp Cell Res 370: 373-382, 2018.

This work is licensed under a Creative Commons Attribution-NonCommercial-NoDerivatives 4.0 International (CC BY-NC-ND 4.0) License. 